\title{
Load-shedding techniques for microgrids: A comprehensive review
}

\author{
Diana Rwegasira ${ }^{\mathrm{a}, \mathrm{b}}$, Imed Ben Dhaou ${ }^{\mathrm{c}, \mathrm{d}}$, Aron Kondoro ${ }^{\mathrm{a}, \mathrm{b}}$, Amleset Kelati ${ }^{\mathrm{a}, \mathrm{e}}$, \\ Nerey Mvungi ${ }^{\mathrm{b}}$, Hannu Tenhunen ${ }^{\mathrm{a}, \mathrm{e}} *$
}

\author{
${ }^{a}$ KTH Royal Institute of Technology in Stockholm, P.O.BOX 229, 16440, Sweden \\ ${ }^{b}$ University of Dar es Salaam, P.O.BOX 33335, 14113, Tanzania \\ ${ }^{c}$ University of Monastir, P.O.BOX 56, 5000, Tunisia \\ ${ }^{d}$ Unaizah College of Engineering, Qassim University, P.O.BOX 6677, Saudi Arabia \\ ${ }^{e}$ University of Turku, P.O.BOX 20014, 20500, Finland
}

\begin{abstract}
The increasing interest in integrating renewable energies source has raised concerns about control operations. The presence of new energy sources, distributed storage, power electronic devices and communication links make a power system's control and monitoring more complex and adaptive than ever before. Recently, the use of agentbased distributed control has seen to have a significant impact on the grid and microgrid controls. The load-shedding technique is among the features used to balance the power consumption in the power system upon less power production. Towards achieving these, different mechanisms, algorithms, challenges, and approaches have been developed and hence need to be reviewed and integrated from the system solution perspective. This research focuses on the review of the state-of-the-art load-shedding techniques, whereby the focus is on control algorithms, simulation platforms and integrations, and control devices for DC microgrid. The research also investigates open issues and challenges that need further investigations. The analyses reported in the paper upholds the importance of the distributed multi-agent system, MAS, in implementing distinct control operations including load-shedding. The effectiveness of the control operations using MAS rely on low-latency and secure communication links in which IoT has been branded as a promising technology for implementing distributed MAS
\end{abstract}

Keywords: Agent Based Systems, Energy management, Load-shedding, multi-agent, smart DC microgrid.

\section{Introduction}

The growing demands for clean and effective electricity system has led to the deployment of Renewable Energy Systems (RES). According to [1], the integration of large-scale RES and storage units in the electric grid reduces the system losses, diminishes the power outage, levels-up the reliability, reduces the carbon footprint and increases the grid efficiency. Other advantages include stable and smart systems which have led to smart grid concept. Fig. 1 shows a conceptual view of a typical smart-grid. Both DC and AC microgrids are considered as pillars in the integration of the renewable energies with the grid.

The smart grid uses intelligent technologies for operations, controls, monitoring and management of the electric grid from the generation side to the end users. These and other attributes have called for the improvement of grid system operations, minimizing human interventions, adding intelligence, etc. The legacy grid system uses Supervisory Control and Data Acquisition System (SCADA) for grid automation.

To facilitate control of the electric grid, an approach of integrating smart devices with agent-based systems, cloud, and fog computing has become more valuable and significant. Due to its virtue in making the grid smart, many designers and developers have started using agent-based systems as a substitute for the SCADA system. According to [2], the agent-based approach with IoT (Internet-of-Things) provides

\footnotetext{
* Manuscript received September 27, 2018; revised March 11, 2019.

Corresponding author. Tel.: +255 767284 666; E-mail address: dianasr@kth.se.

doi: $10.12720 /$ sgce.8.3.341-353
} 
scalability, mobility and secure communications on scheduling problems as well as operational issues. The authors of [3] used IoT spots to embed some devices which include smart sensors, actuators, Raspberry Pi and Arduino for microgrid processes which further facilitates the automatic functionalities into the system. The smart sensors and actuators act as agents to provide signals and actions into the systems with scant or no human interventions, the Arduino for connections and interactions between components and Raspberry Pi for processing activities. The results of combining agents and cloud paradigms led into enhanced stability and management of a distributed real-time system. These have also led to different monitoring activities on electricity such as self-healing, fault detection and load-shedding techniques. Other places which have adopted the use of multi-agent include health systems, transport systems and telecommunications.

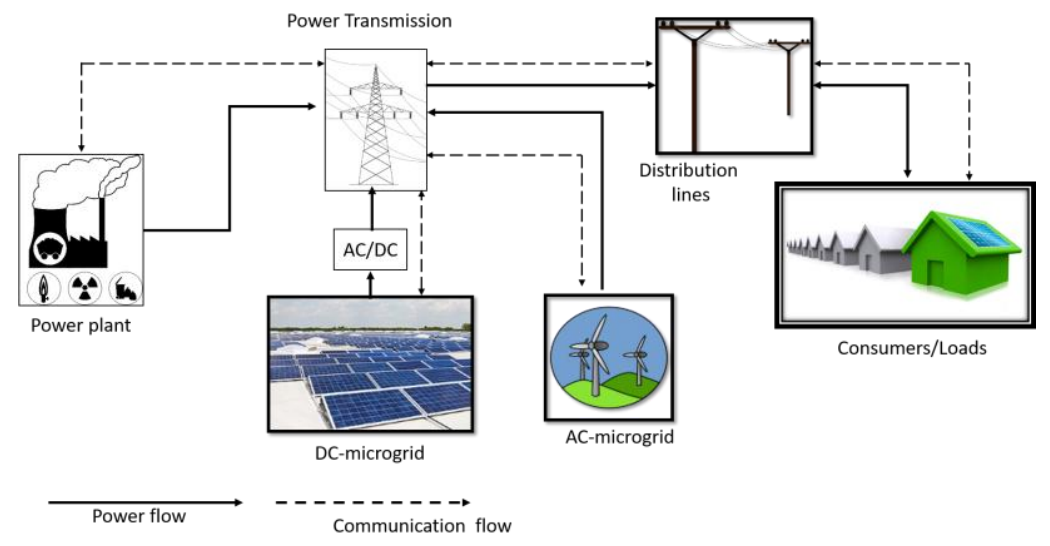

Fig. 1. Overview of a smart-grid with distributed microgrid

Demand-Response (DR) capabilities are considered as a revolution in the operation of the grid. The DR program consists of matching demands to responses using three variant techniques: Peak clipping, valley filling, and load-shifting [4].

Load-shedding/shifting technique opts to shift the operations of non-critical appliances to off-peaks. The work of [5] discussed the load-shedding technique with different perspectives. Two types of architectures are used, i.e. centralized and decentralized (distributed), where centralized approach focuses on the central control systems and the decentralized approach focuses on an individual component works and resource sharing based on the demand.

This paper reviews the recent load-shedding algorithms used in the process of control and monitoring of the electric grid and microgrid systems. The paper also discusses the implementation and challenges of the platforms as well as open issues. The rest of the paper is organized as follows: Section 2 elaborates the rationale on the load-shedding process with respect to our work. Section 3 discusses the control operations of load-shedding which includes optimization process and algorithms, platforms. Section 4 discusses multi-agent systems for load-shedding with their strategies. Section 5 discussed the challenges and open issues with regards to load-shedding while Section 6 concludes the work.

\section{A Conceptual Framework for Load-shedding}

Demand-Response program is an advanced feature supported by the smart-grid. The program aims at, among others, improving the efficiency of the grid, reducing the electricity bill, lowering the operating cost, and combating carbon dioxide emission. Load-shedding (LS) is an effective technique for the realization of the demand-response program. LS aims at matching demands and supplies under various objectives. In the context of smart-grid with distributed energy resources load-shedding has been used for different responses such as reduces the interruption cost at the feeders, insures voltage stability, stabilizes the voltage and frequency in the power system, improves the reliability of the distribution system, restores 
services in distribution network, and prevents cascading failure [6]. Demand-Response program can be incentive-based or pricing-based as depicted in Fig. 2 [7].

Load-shedding is a control strategy that can be executed either by the utility company or by the consumer in a bid to operate an optimum number of devices/ appliances at a given time interval. Loadshedding is an incentive-based program that fosters users to switch off non-critical appliances during peak hours. In residential settings, advanced-metering infrastructure provides two-way communication between the consumer and the utility companies. In AMI, the load-shedding program is instigated using a smart-meter.

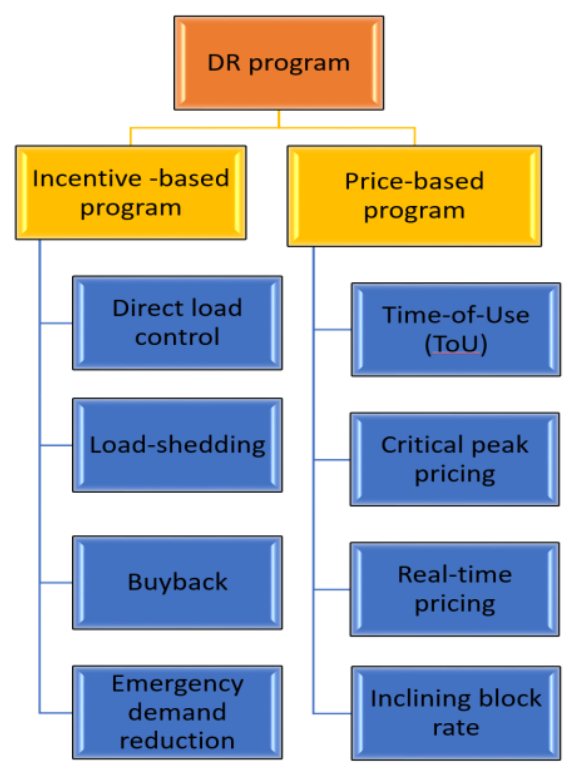

Fig. 2. Categorization of DR programs

\section{DC Microgrid}

A microgrid is a cluster of distributed loads (e.g. Houses, Electric Vehicles), distributed energy storage devices (e.g. Lithium batteries) and distributed generation sources (e.g. Solar and wind power sources). It is capable of operating either in grid-connected mode or island mode with the purpose of supplying reliably low voltage electricity in the community. Two commonly control strategies are Power Quality control, PQ, and Voltage, Frequency control, FV, which can be used in microgrid operations [8]. Power quality focuses on the control of active/reactive power, back-to-back converter, real-time self-tuning while VF focuses on the smooth mode transition from grid connected to islanded mode [9].

In recent years, researchers have paid significant attention to the DC microgrid compared to AC microgrid. Literally, DC microgrid has numerous advantages, such as, lower conversion loss and transmission loss, less complex control strategy, and higher quality electric power system[10]. For DC microgrid, only P-V droop control methods are used.

Smart DC microgrid is a recent technology which has risen due to the following reasons: (i) can be disconnected for the grid in an advent of fault, (ii) supports the integration of distributed generators (iii) enhances stability and reliability of the system, (iv) helps reducing greenhouse emission, (iv) improves power quality and (v) saves the cost of energy source [11]. The control methods of smart DC microgrid can be divided into two categories namely: centralized and distributed control. On a regular basis, distributed control is best achieved due to less reliance on the communication system and with three types of control strategies: droop control, hierarchical control and DC bus signaling [12]. Fig. 3 describes the nature of the smart DC microgrid with its components. 
The level of controlling and monitoring in microgrid depends on different aspects incorporated such as latency, power consumption, security issues and power management. Different algorithms, techniques and frameworks have been developed to account for these behaviors. The research in [13] introduced agent-oriented analysis and design model known as Gaia methodology for multi-agent systems to deal with both the macro-level (societal) and micro-level (agent) aspects. GridSpice platform was developed to enable large electric network modeling to connect the boundaries between generation, transmission, distribution and customers [14]. The platform can be used in the Distributed Management Systems (DMSs) for control and monitoring purposes as well as to optimize the scheduling dispatch for flexible loads.

DC microgrid is getting popular as it requires less complicated control and management techniques, among other feature. Furthermore, the generation of energy from renewable sources can be done at the customer premises.

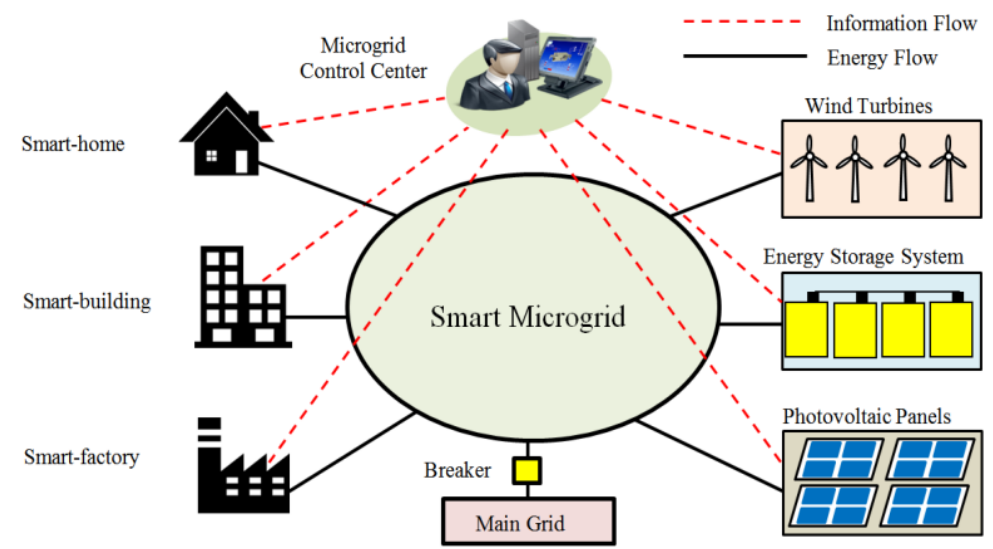

Fig. 3. smart microgrid control and monitoring system

\section{Control Operations for Load-shedding Technique}

In electric grid systems, control algorithms need communication for efficient operations, autonomous, and automatic actions. This has been triggered through better control operations in terms of voltage and frequency regulation, proper load sharing and coordination in the distributed energy resources, power flow control between the microgrid and the main grid, the operating cost optimization and synchronization of microgrid with the main grid. Other factors can be based on cost reduction and energy saving in the applied system. These are aspects which normally contribute in the load-shedding processes using different strategies such as multi-agent systems, fuzzy logic and neural networks.

\subsection{Taxonomy of load-shedding techniques}

In modern building, the balancing between the power demand on the appliances and the available power is significant. Different techniques have been introduced as described in Fig.4 based on Loadshedding which have advantages, disadvantages as well as the applicability in the power system [15]. In many cases Adaptive Load-shedding (ALS) is mixed with Conventional Load-shedding (CLS) because of frequency and voltage control in the latter method and adaptation capability in the former method. The good example on the mixed approach is the use of ROCOF relay (Rate of Change of Frequency) upon time derivatives and frequency regulation of the power system. ALS is the best with the Markov Chain Monte Carlos methods due to its randomized optimization process when implementing in the primary control. The CLS is mostly applicable on the Model Predictive Control as it can work in offline mode systems. The technique normally defines layers of Finite State Machine (FSM) which can be improved with timers especially on sensors and actuators. For the sensitive systems, the most advised technique to be used is the Computational Intelligent Load-shedding (CILS). The CILS is accurate and requires to work in online state hence provides optimal results. 
The convenient technique in the load-shedding control is the ALS thanks to its capability in adjusting the decision criteria, the autonomous behavior, etc. Those features made him fit in the self-healing process. The CILS technique has been proven to be the best due to their robustness and flexibility with complex non-linear and large power systems. However, using this technique slow the system upon simulation because several steps should be done before such as: faults in the system, instabilities of the system, testing of each components, etc. CLS are limited on achieving optimum load-shedding because of failure to estimate the accurate amount of power imbalance in the systems although they can control frequency and voltage simultaneously [16]. At the end of the system, the most and intended output is to have the optimum load-shedding while maintaining the power systems stability and make the complete system safe, secure and fast.

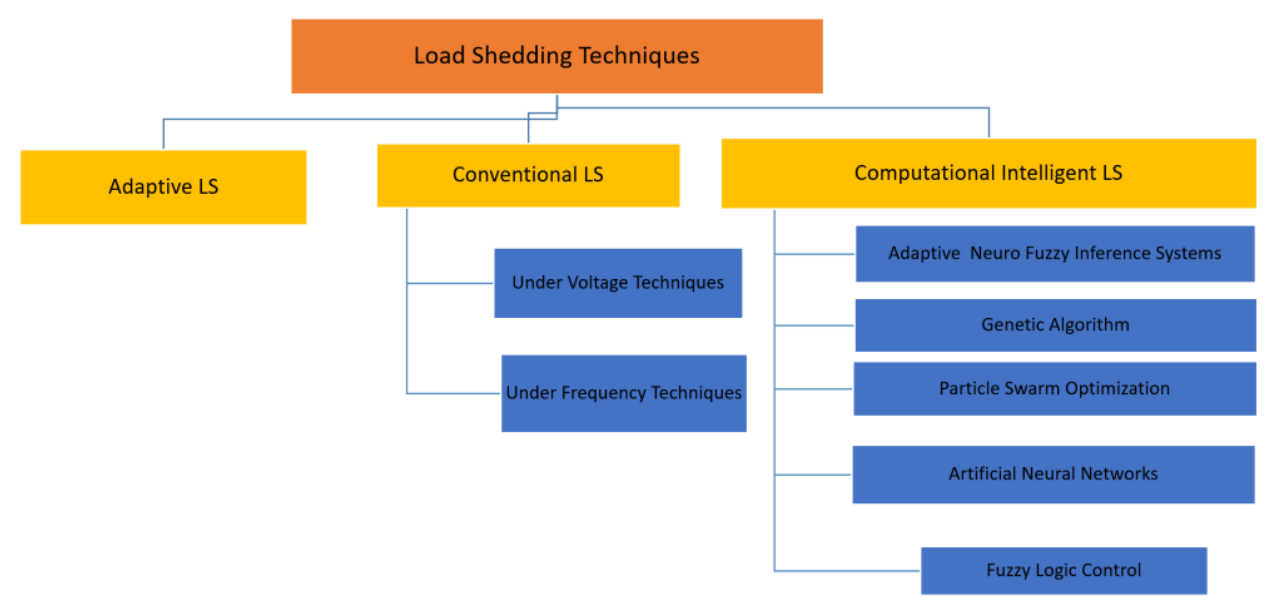

Fig. 4. Load-shedding technique categories [15]

The efficiency of the system depends on different factors such as the algorithm, security mechanism, communication topologies and adaptability upon changes. In load-shedding, several studies have worked on different types of algorithms as listed in Table 1 to achieve optimization process. Majority of works show that load-shedding can be geared towards its implementation. One of the most useful tool is Matlab software for mathematical calculations. The focal points in the load-shedding achievement are the control and monitoring of several grid parameters such as frequency, voltage, reactive power, storage batteries, etc. [17]. The selection of optimization process depends on the target outputs, usefulness and implementation complexity. Apart from software simulations, load-shedding can be done with smart devices such as smart sensors, microcontrollers, and actuators.

Table 1. Comparison of optimization process on load-shedding techniques

\begin{tabular}{|l|l|l|l|}
\hline Optimization process & LS technique & Work & Simulation Environment \\
\hline Ant-Colony Optimization & Adaptive & {$[16],[18]$} & \multirow{2}{*}{ Matlab, PSCAD, dSPACE } \\
1103 board, \\
Java 8, IEEE bus systems, \\
Controllers (PLC)
\end{tabular}




\subsection{Fundamentals of load-shedding technique}

The control of load-shedding is the contribution of several components and steps including algorithm, flow chart, procedures and the used simulation platforms. The main procedures of the load-shedding are supported by some relative equations [25]:

i. Define the objective functions (L) of decision variables (x). This can represent what is the aim of the shedding process such as supply and demand in the systems or power flow of the model and variables can represent houses, source of power, appliances, etc. Equation 1 present the summation of the load-shedding $\mathrm{L}$ in the $\mathrm{n}$ houses with $\mathrm{D}$ as a set of demand loads and equation 2 is for load-shedding for each demand site

$$
\begin{gathered}
L=\sum_{n=1}^{X}\left(l_{n}\right), \forall n \in D \\
l_{n}=\sum_{n=1}^{X}\left(Y_{n, m}\right) \leq 0, \forall n \in D
\end{gathered}
$$

ii. Define the linear inequality constraints matrix (b) with vector (v). In this case, the definition of different operational modes such as on-state/off-state, critical and non-critical loads are considered. Equation 3 presents the set of constraints $C$ on each variable Y.

$$
\left|\mathrm{Y}_{\mathrm{n}, \mathrm{m}}\right| \leq C_{n, m}
$$

iii. Define the set of lower and upper boundaries for decision variables such as time, price, power consumptions, etc.

iv. Define the decisions and action to be made before, during and after load-shedding process at each time. Example equation 4 calculate the amount of load-shedding LD to be shed for maintaining the minimum frequency for maximum anticipated overload. $\mathrm{L}$ is the unit overload, fmin is the minimum permissible frequency. $\mathrm{D}$ is the load reduction factor and $\mathrm{fn}$ is the nominal frequency.

$$
L D=\frac{\frac{\mathrm{L}}{1+\mathrm{L}}-\mathrm{d}(1-(\mathrm{fmin}-\mathrm{fn}))}{1-\mathrm{d}\left(1-\left(\frac{\mathrm{fmin}}{\mathrm{fn}}\right)\right)}
$$

The control and monitoring of load-shedding has moved towards the use of agent-based systems. This has been realized due to the advantages and features of agents in the power grid applications. The previous applications were missing the attributes including mobility, autonomous, sociability, adaptability and dynamicity [27].

\section{Load-shedding with Multi-Agent Systems}

The use of automation systems has become more useful nowadays for life simplification and error reduction. The applications of agent-based systems are important due to properties such as mobility, cooperative, communicative and learnable. Other properties are autonomous, reactive, pro-activeness and social ability. These features are more important in our daily lives to reduce human interactions. Some advantages mentioned in [28] for agent-based systems include reducing the network load, minimizing the network latency, tolerating faults and executing in asynchronous and autonomous mode. The main components on forming agent-based control for microgrid includes: Energy source unit, energy storage unit, load, energy source agent, energy storage agent, HMI (Human Machine Interface) and load agent, [29] or as power plant agent (PA), substation agent (SA) and coordinator agent (CA) [19]. An agent can 
either be physical a entity which refers to the natural environment or virtual entity for software and database.

The use of agent-based systems especially in the electric power field provides a natural approach for studies, modeling and simulation of the smart microgrid. Advantages of MAS include the ability to survive single-point-failures and decentralized data processing, which leads to efficient task distribution, eventually causing faster operation and decision-making process. In artificial intelligence, agent-based technology has been hailed as a promising paradigm for conceptualizing, designing, and implementing software systems [26]. Agent-based systems emphasize the use of smart devices with IoT, control of the electric grid in a smart manner as well as simulation capabilities, [5]. These features bring the concern also on a load-shedding process, self-resilience, fault detection, etc. upon utilizing the agent-based technology as it brings flexibility, adaptability, reliability and energy management in the electric grid.

Many researchers have solved load-shedding problems using Multi-agent Systems (MAS) as one of the recentl best solutions [21]. The most common approach is based on the decentralized multi-agent systems with computational paradigm [30]. For load-shedding, agent framework consists of hardware part, such as sensors and actuators, and software part, such as agent communication layers, which are Foundation for Intelligent Agents (FIPA) and Knowledge Query Multiplication language (KQML). Fig. 5 depicts the load-shedding program using multi-agent systems. In this manner, the control units are involved with algorithms and logic towards the achievement of load-shedding technique. The taken action has to be fed into the data concentrator to effect on the processor. The processor links the hardware and software part for manipulation. The actuators are the outcomes after the load-shedding process has been done. The advantages of this framework are to keep on monitoring the system and also make the use of real-time data, which is significant in the smart grid systems. This can be done with the help of IoT devices which is also the basic feature of the integrated and embedded systems.

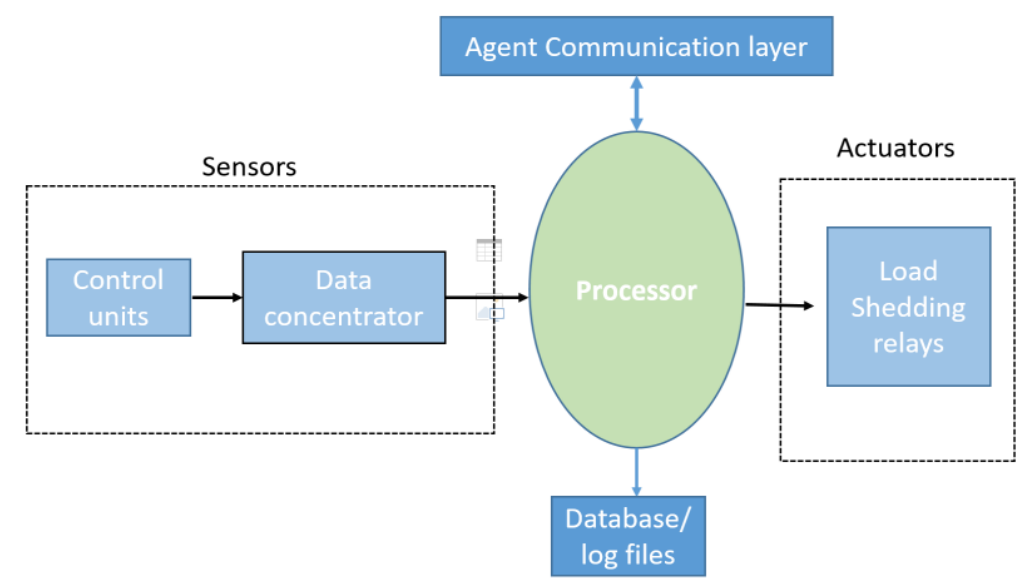

Fig. 5. Agents framework for load-shedding [30]

Working with multi-agent systems requires simulation platforms for visualization purposes. Different platforms for MAS applications have been described in [31] with explanations on their design, implementations and usage. Many platforms are java based language which makes the platform independent. Recently, new control applications are focusing on the integration of two or more platforms for better optimization and outputs visualizations. The works of [32] and [33] integrate Jade and Repast platforms for MAS in the application of agent behavior components and complex simulation, respectively. The selections of these tools are due to the step-by-step simulations in Repast and behavior formulation in Jade. In [34], the authors combined Matlab and Zeus for three purposes: (i) Zeus for building MAS (ii) Matlab for modeling the microgrid (iii) TCP/IP socket interface for connections between agents and microgrid. Moreover, the load-shedding framework with hardware-in-loop, HiL, has been developed using Raspberry pi, Jade and Repast platforms to provide flexibility of the simulations and realization of 
the real systems [35]. As load-shedding requires real-time actions, therefore Jade and Repast are the best platforms to use. Generally, these frameworks can be applied to any controlling and monitoring applications such as self-healing and fault detection. Fig. 6 shows the integration architecture platform with Jade and Repast tools and how Jade is integrated with microcontrollers with solar driven DC microgrid.

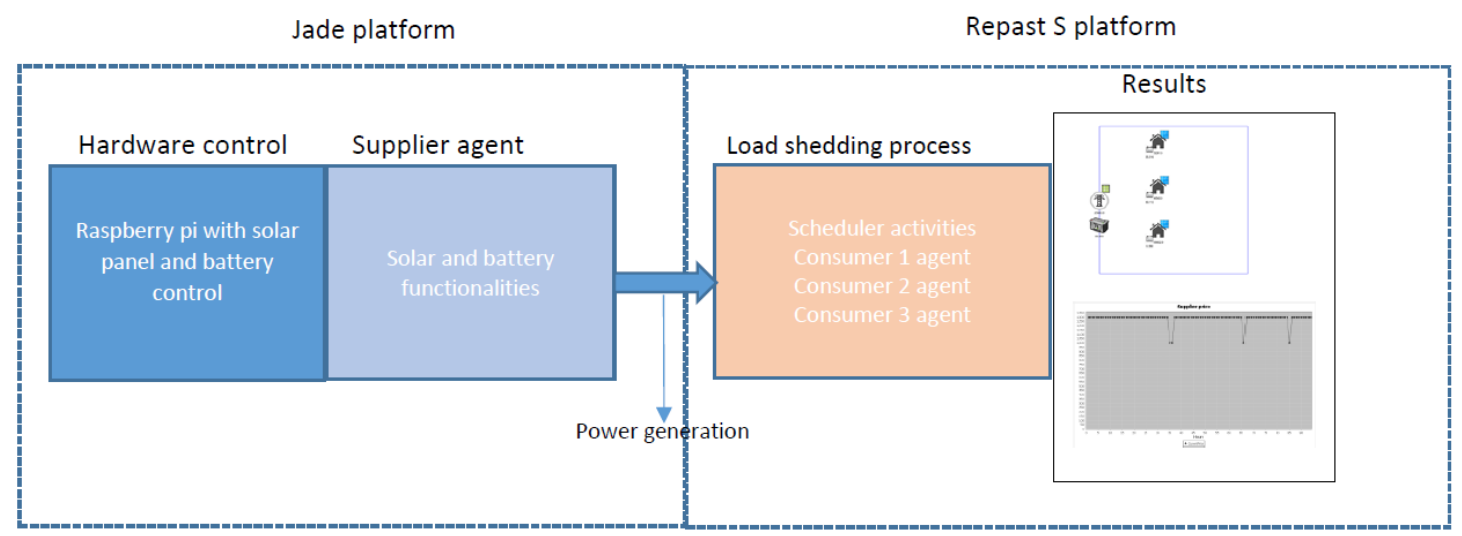

Fig. 6. Hardware in loop for load-shedding using multi-agent

Several research works have worked on load-shedding technique with multi-agent as summarized in Table 2. The decentralized approach has worked successfully as it provides the flexibility of each agent to adapt the environment and act accordingly. In this manner, it is easy for an individual agent to make its own decisions on its own rather than waiting for the central agent to do. Table 3 categorizes the simulation platforms according to their attributes and behavior towards the use of multi-agent systems. Fig. 7 provides the flowchart to follow upon using a multi-agent system for load-shedding technique.

Table 2: Load-shedding process using MAS

\begin{tabular}{|l|l|c|}
\hline Agents state & Work & Simulation Environment \\
\hline \multirow{4}{*}{ Centralized } & {$[26]$} & IEEE bus systems \\
\cline { 2 - 3 } & {$[19],[20]$} & Matlab \\
\hline \multirow{3}{*}{ Distributed/Decentralized } & {$[21][22][36]$} & Jade \\
\cline { 2 - 3 } & {$[37][38][39]$} & Repast Simpony \\
\cline { 2 - 3 } & {$[40],[34][41]$} & Matlab,Zeus, PSCAD \\
\hline
\end{tabular}

Table 3: Comparison of simulation platforms

\begin{tabular}{|c|c|c|}
\hline Attribute & Best platform & Reason \\
\hline Simulation framework & Repast & $\begin{array}{l}\text { - Step by step simulation, } \\
\text { - Have scheduler method }\end{array}$ \\
\hline Device integration & \multirow[b]{2}{*}{ Jade } & - $\quad$ FIPA Agent Communication language for physical agents. \\
\hline Dynamic topology & & - $\quad$ Distributed applications composed of autonomous entities \\
\hline Interaction & Matlab & $\begin{array}{l}\text { - For mathematical computations } \\
\text { - } \quad \text { Many platforms have included the capability to interact with it }\end{array}$ \\
\hline Ontologies & Jade & - Domain knowledge and physical representation, \\
\hline Communication & Jade & - $\quad$ Agent Communication language \\
\hline
\end{tabular}




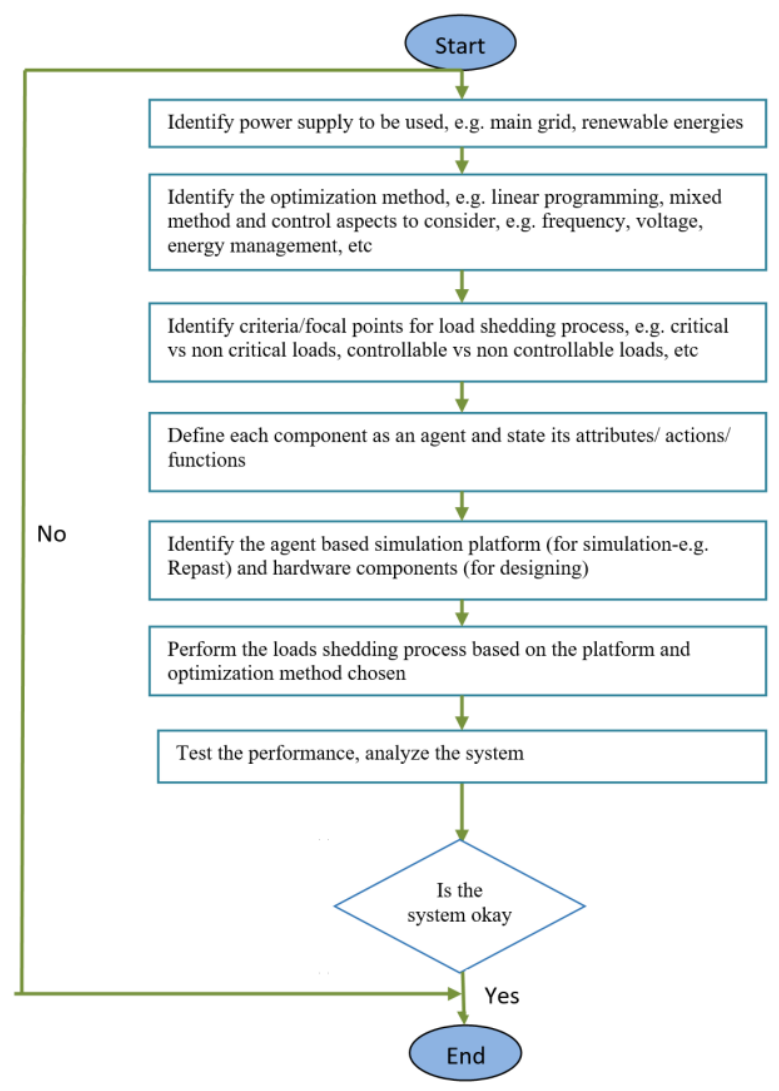

Fig. 7. Flow chart for load-shedding with multi-agent systems

\section{Scenario for Pricing and Energy Cost}

In this paper, we will demonstrate the benefit of a dynamic pricing scheme to save energy costs using typical consumer data. Consider a consumer having several appliances such as a washing machine, dishwasher, clothes dryer, each having its own power consumption upon usage. Also, assume that the customer spends 120 minutes per week on washing and drying the clothes with the washing and dryer machines during the winter, fall and springtime. The power consumption of both appliances in total is $5 \mathrm{~kW}$ upon usage. With respect to this scenario, it was decided to use the Power Smart Pricing program, available in [42]. Its basic function is to provide daily information on the energy price to the consumer. In this case, the customer may use an online website company, the registered phone or an app to get the current hourly power price of the next day.

Taking the data shown on Fig.8, the comparison of the price and be elaborated. For example, at 4 a.m. the price is 2.0 cents $/ \mathrm{kWh}$ while at $4 \mathrm{p} . \mathrm{m}$. the price is $4.5 \mathrm{cents} / \mathrm{kWh}$. With this fact, the consumer will spend 20c per day at 4 a.m. and $45 \mathrm{c}$ per day at 4 p.m., respectively. Assuming the cost remains the same throughout the year, the household will spend $1040 \$$ when washing clothes at 4 a.m. and $2340 \$$ at 4 p.m. In this case, a total amount of $1300 \$$ will be saved by using this smart pricing program. The advantage of this application is to give feedback on a real-time basis and provide the adjustment accordingly to the consumer side. This can also be seen in [43] regarding real-time demand response model developed.

With this process, the electric company can decide to shift the activities for industries in the middle time and leave the morning hours for consumers and hence make the pricing factor realistic (price based demand response). Consumers will also be able to save cost when planning to use that scheduling time for 
washing machines. The study in [44] applied the same approach on the demand response through load scheduling and power shifting activities management. These serve the purposes for energy saving, electric market control, resource management as well as system stabilization.

Current Hourly Prices for May 21, 2018

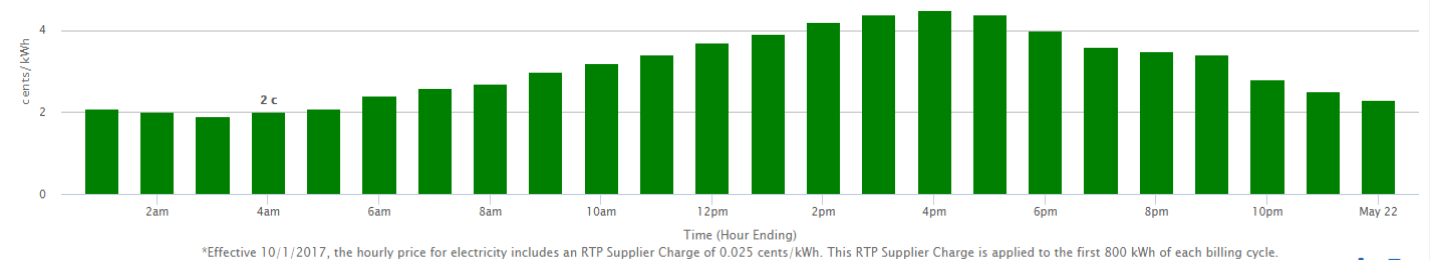

Fig. 8. Power pricing

The concept of optimization technique and demand responses in the load-shedding process can be improved through the involvement of technologies such as agent-based systems whereby each agent (consumer, generation and transmission) can detect the actions and make changes based on the conditions and attributes given in the systems. Agents are more intelligent and easy to adapt based on the environments hence the control mechanism will be achieved. The control and monitoring processes are more accurate and fast upon implementation using agents.

\section{Discussion and Open Issues}

Control and monitoring processes in the electric power system are fundamental aspects to facilitate the integration of smart microgrid. To master these attributes, different systems and mechanisms are required in handling activities from distribution, transmission to end-user side of the electrical network. The loadshedding process is one of the demand responses which makes use of demand and supply in the community so as to avoid the blackout of the complete system [45][46]. The operation of load-shedding in the DC microgrid is still an open issue which needs the research through considering attributes such as pricing with hour-ahead notification, real-time control, monitoring and notification. The advantage of the power stability in DC microgrid will lead to the stability of the system hence the optimization process will be stabilized as well.

In the management of distributed energy systems, agent-based systems have been seen to work successfully. However, control and optimization processes in the consumer side have been mentioned as a challenge by different researchers [46], [47]. This involves the interconnections between distributed plant, centralized controller and sensors for transmission of information which all have to be in a network for communication, pricing issues, PAR, etc. The task of optimization has also remained as a concern to the majority of the designers and users due to algorithms involved and demand responses to take into account. Moreover, the behavior towards the action of agents in terms of self-organization in the operations of distributed designs is still a challenge [47]. Moreover, in terms of large number of premises, the control actions on distributed energy resources is more difficult,[47]. In some cases, human intervention is needed to control the distribution and transmission parts, especially when integrating with renewable energy resources. Other open issues need further research based on the control using multi-agent systems on the system include frequency regulation and balancing multiple generators, optimal robust control in solar irradiance level, algorithm on hybrid control for determining the state of energy storage and solar radiation expectation.

Decentralized demand-response program with energy sharing among users is also an open issue where individuals (multi-agents) can contribute to the production of power/energy in the distribution systems. The electric companies and stakeholders need to focus on the energy management whereby an individual can sell extra power he/she has in the electric grid rather than the main grid to supply to all consumers.

The idea is to use fuzzy logic control method which will control and monitor the consumption and 
usage of each consumer. However, the centralized approach can achieve the same performance but with an additional cost due to the fact that one will need the central control to mobilize and communicate with all nodes upon topology changes. With respect to demand and cost optimizations, things like emission, fuel availability and weather conditions may affect the price of electricity in the market.

One of the advantages of the simulation is to obtain the initial and considerable useful information in the implementation of the real-time systems with features such as pricing, critical peaks and system usage time. These require much better understanding and exploitation of the concepts in smart grid [48]. In smart grid, the selection of which platform to use in the simulation of the multi-agent system with respect to load-shedding is still a challenge to the designer. Despite the fact that there are many open source platforms but to work with load-shedding need further discussions as it involves the customers' satisfaction as well as demand response [21]. So far, agent-based simulation is the best technology in providing realistic and readily validation means of evaluating the performance in smart grid functions [49]. Load-shedding using the evolutionary algorithm is widely used for addressing the problems.

Bidirectional communication with the electric supplier in smart grid needs a design procedure for interaction to take place and energy minimization in the idle time of use. The experience shows that the hourly basis algorithm to monitor the consumption will save the purpose [43] though the internet connections and bandwidth might be a challenge in the developing countries. Additionally, security attacks and cost implications are areas of concern that need further investigations. IoT is another technology which has now emerged in smart grid with the use of multi-agent systems. Chiu in [50] discusses IoT against demand-side management implementations. This relies on formulating dynamic pricing model for demand-side management. To integrate this with renewable energies still, need further investigations.

Agent Communication Languages and Ontologies are also major concerns in the smart grid. So far it is only the Jade platform which has the ACL though it does not provide the simulator capabilities. Therefore, to work with Jade one needs also the integration of other platforms for simulation purposes. Furthermore, to interact with platforms one needs basic skills especially on the programming languages and mathematics as well. To facilitate the development and testing new load-shedding algorithms, both physical agents and multi-agent simulation platform need to be integrated (HiL). This path of research needs further attention.

\section{Conclusion}

For future intelligent microgrid systems, the need of deploying smart devices in the controlling and monitoring activities is significant due to the fact that, the system requires real-time synchronization, stability, and quality power, secure communication, hour-ahead pricing notification, etc. Based on the growing demand on the efficiency and reliable power, the integration of distributed renewable energies in the smart-grid addressed the power shortage, improved the reliability of the grid, trimmed the cost, etc. Demand-response, DR, programs can further shave the high-peaks, reduces the failure rate, among others. Load-shedding for DR program has proven to be the key program in the demand-side management. Moreover, using multi-agent systems in microgrid provide flexibility of solving the problems in the electric power system which is beyond the capabilities of a single agent. For load-shedding technique the application of multi-agent systems has worked well and more efficiency. In this paper, we have surveyed state of the art research in the load-shedding techniques for the smart-grid with a potential overview of the system, advantages and disadvantages, challenges and open issues. The work provided a clear understanding of the load-shedding process as well as pinpointed the basic algorithms required in the implementation of it. The research further focused on the microgrid systems to explore the challenges towards designing and implementation of intelligent load-shedding schemes. Load-shedding for a DCmicrogrid has been identified as a topic that needs further investigations by the research communities. Furthermore, the impact of communication on the reliability of the load-shedding techniques needs more work. 


\section{Acknowledgment}

This work is supported by Swedish government through the SIDA project. Much thanks also go to Dr. Masoumeh Ebrahimi of Royal Institute of Technology, KTH for her support on the improvement of the work and grammatical error corrections.

\section{Reference}

[1] Sarkar J and Bhattacharyya S. Operating characteristics of transcritical CO2 heat pump for simultaneous water cooling and heating. Arch. Thermodyn., 2012; 33(4): 23-40.

[2] Ansola PG, Higuera AG, Otamendi FJ, De Morenas J. Agent-Based Distributed Control for Improving Complex Resource Scheduling: Application to Airport Ground Handling Operations. 2014; 8(4): 1145-1157.

[3] Fortino G, Guerrieri A, Russo W, and Savaglio C. Integration of agent-based and Cloud Computing for the smart objectsoriented IoT. in Proceedings of the 2014 IEEE 18th International Conference on Computer Supported Cooperative Work in Design, CSCWD 2014, 2014: 493-498.

[4] Nunna HSVSK, Saklani AM., Sesetti A, Battula S, Doolla S, and Srinivasan D. Multi-agent based Demand Response management system for combined operation of smart microgrids. Sustain. Energy, Grids Networks, 2016; 6: $25-34$.

[5] Mortaji H, Ow SH, Moghavvemi M, Almurib HAF. Load-shedding and Smart-Direct Load Control Using Internet of Things in Smart Grid Demand Response Management. IEEE Trans. Ind. Appl., 2017; 53(6): 1-1.

[6] Shokooh F. et al., Intelligent load-shedding case study of the application in a large industrial facility. IEEE Ind. Appl. Mag., 2011; 17(2): 44-53.

[7] Deng R, Yang Z, Chow M. A survey on demand response in smart grids : Mathematical models and approaches, IEEE Trans. Ind. Informatics, 2015; 11(3): 570-582.

[8] Hajimiragha AH, Zadeh MRD. Research and development of a microgrid control and monitoring system for the remote community of Bella Coola: Challenges, solutions, achievements and lessons learned. IEEE Int. Conf. Smart Energy Grid Eng. SEGE 2013, pp. 1-6, 2013.

[9] Venkatraman R and Khaitan SK. A survey of techniques for designing and managing microgrids. IEEE Power and Energy Society General Meeting. 2015: 1-5.

[10] Gulin M. Control of a DC Microgrid, University of Zagreb, 2014.

[11] Kurohane K, Senjyu T. A Hybrid Smart AC / DC Power System. IEEE Trans. Smart Grid, 2010; 1(2): 199-204.

[12] Wen H. and Zhu W. Control and Protection of DC Microgird with Battery Energy Storage System. in IEEE International Conference on Power Electronics, Drives and Energy Systems, PEDES, 2016: 1-6.

[13] Davoodi SF, Tang WH, and Wu QH. Implementation of Gaia methodology for multi-agent based transformer condition monitoring. in IEEE PES Innovative Smart Grid Technologies Conference Europe, 2012, pp. 1-8.

[14] Anderson K, Du J, Narayan A, and Gamal AEl, "GridSpice : A Distributed Simulation Platform for the Smart Grid," in IEEE Transactions on Industrial Informatics, 2014, vol. 10, no. 4, pp. 2354-2363.

[15] Laghari JA, Mokhlis H, Bakar AHA, and Mohamad H. Application of computational intelligence techniques for loadshedding in power systems: A review. Energy Convers. Manag., 2013; 75(1): 130-140.

[16] Hasanat M, Hasan M, Ahmed I, Chowdhury MI, Ferdous J, Shatabda S. An ant colony optimization algorithm for loadshedding minimization in smart grids. in 5th International Conference on Informatics, Electronics and Vision (ICIEV), 2016: 176-181.

[17] Terzija VV. Adaptive underfrequency load-shedding based on the magnitude of the disturbance estimation. IEEE Trans. Power Syst., vol. 21, no. 3, pp. 1260-1266, 2006.

[18] Cepeda J. C., Ramirez D., and Colome D. G., "Real-time adaptive load-shedding based on probabilistic overload estimation," in 2014 IEEE PES Transmission \& Distribution Conference and Exposition - Latin America (PES T\&D-LA), 2014, no. September 2016, pp. 1-6.

[19] Xie J., Liu C. C., and Sforna M., "Distributed underfrequency load-shedding using a multi-agent system," in 2015 IEEE Eindhoven PowerTech, PowerTech 2015, 2015, pp. 1-6.

[20] Ghorbani M. J., Choudhry M. A., and Feliachi A., "Distributed Multi-Agent based Load-shedding in Power Distribution Systems," 2014 IEEE 27th Can. Conf. Electr. Comput. Eng., pp. 1-6, 2014.

[21] Logenthiran T., Srinivasan D., and Khambadkone A. M., "Multi-agent system for energy resource scheduling of integrated microgrids in a distributed system. Electr. Power Syst. Res., 2011; 81(1):138-148.

[22] Choi Y, Lim Y, and Kim HM. Optimal load-shedding for maximizing satisfaction in an Islanded microgrid. Energies, 2017; 10(1): 1-13.

[23] Khoa TD, Dos Santos LT, Sechilariu M, and Locment F. Load-shedding and restoration real-time optimization for DC microgrid power balancing. in 2016 IEEE International Energy Conference, ENERGYCON 2016, 2016: 1-6.

[24] Trigueiro dos Santos L., Sechilariu M., and Locment F. Optimized Load-shedding Approach for Grid-Connected DC Microgrid Systems under Realistic Constraints. Buildings, 2016; 6(4): 50-65.

[25] Seyedi H. and Sanaye-Pasand M. New centralised adaptive load-shedding algorithms to mitigate power system blackouts. Gener. Transm. Distrib. IET, 2007; 1(2): 1-16.

[26] Xu Y., Liu W., Gong J., Stable multi-agent-based load-shedding algorithm for power systems. IEEE Trans. Power Syst., 2011; 26(4): 2006-2014.

[27] Engler A, Hardt C., Jimeno J., Ruela J, Oyarzabal J, “Agent based micro grid management system," Int. Conf. Futur. Power Syst. Novemb., pp. 1-5, 2005. 
[28] Thomas MV and Sekaran KC. Agent-Based Approach for Distributed Access Control in Cloud Environments. in International Conference on Advances in Compuing,Communications and Informatics (ICACCI), 2013:1628-1633.

[29] Merabet GH. et al. Applications of multi-agent systems in smart grids: A survey. in International Conference on Multimedia Computing and Systems (ICMCS), 2014; 1088-1094.

[30] Xie J, Liu CC, and Sforna M. Agent-based distributed underfrequency load-shedding. 2017 19th Int. Conf. Intell. Syst. Appl. to Power Syst. ISAP 2017, 1-6, 2017.

[31] Kravari K. and Bassiliades N. A Survey of Agent Platforms. Journal of Artificial Societies and Social Simulation, 2015. [Online]. Available: http://jasss.soc.surrey.ac.uk/18/1/11.html. [Accessed: 28-May-2018].

[32] Yoo MJ and Glardon R. Combining JADE and repast for the complex simulation of enterprise value-adding networks. Lect. Notes Comput. Sci. (including Subser. Lect. Notes Artif. Intell. Lect. Notes Bioinformatics), 2009; 5386: 116-130.

[33] Görmer J, Homoceanu G, Mumme C, Huhn M, and Müller JP. JREP: Extending Repast Simphony for JADE agent behavior components," Proc. - 2011 IEEE/WIC/ACM Int. Conf. Intell. Agent Technol. IAT 2011, 2011; 2: 149-154.

[34] Xiao Z. et al., Hierarchical MAS based control strategy for microgrid. Energies, 2010; 3(9): 1622-1638.

[35] Rwegasira D, Ben DI, Kondoro A, Kelati A, Mvungi N, and Tenhunen H. A Hardware-in-Loop Simulation of DC Microgrid using Multi-Agent Systems. in 22nd CONFERENCE OF FRUCT ASSOCIATION, pp. 3-8.

[36] Lim Y, Kim HM, and Kinoshita T. Distributed load-shedding system for agent-based autonomous microgrid operations. Energies, 2014; 7(1): 385-401.

[37] Rwegasira D. et al., A framework for laod shedding and demand response in DC microgrid using multiagent system. in 201721 st conference of Open Innovations Association (FRUCT), 2017, pp. 284-289.

[38] Rwegasira D, Dhaou IB, Kondoro A., Shililiandumi N., Kelati A., and Mvungi N. A Multi-Agent System for Solar Driven DC Microgrid. in International Conference on Control, Electronics, Renewable Energy and Communications (ICCREC), 2017, pp. 252-257.

[39] Xu Y, Liu CC, Chen Y, and Gao H. Dynamic load-shedding for an islanded microgrid with limited generation resources. IET Gener. Transm. Distrib., 2016; 10(12): 2953-2961.

[40] Wu X, Feng S, and Jiang P. Distributed coordination load-shedding of islanded microgrids based on sub-gradient algorithm. IEEE Access, 2017; 5(1): 27879-27886.

[41] Liu W. et al., Improved average consensus algorithm based distributed cost optimization for loading shedding of autonomous microgrids, Int. J. Electr. Power Energy Syst., 2015; 73: 89-96.

[42] Ameren I, "Powe Smart Pricing," Ameren Illinios, 2018. [Online]. Available: https://www.powersmartpricing.org/. [Accessed: 28-May-2018].

[43] Conejo AJ, Morales JM, and Baringo L. Real-Time Demand Response Model. Smart Grid, IEEE Trans., vol. 1, no. 3, pp. 236-242, 2010.

[44] Vardakas JS, Zorba N, and Verikoukis CV, A Survey on Demand Response in Smart Grids: Pricing Methods and Optimization Algorithms. IEEE Trans. Ind. Informatics, vol. 11, no. 3, pp. 1-1, 2015.

[45] Tomar KKS, and Singh SN. Energy management and control of microgrid using multi-agent systems. 2015 IEEE UP Sect. Conf. Electr. Comput. Electron. UPCON 2015, 2015.

[46] Ciara B, The D, Engineering M, Supervisor P, and Duignan R. Modelling Demand Response in the Residential Sector, University College Dublin, 2011.

[47] Lequay V. et al., Flexible load-shedding using gossip communication in a multi-agents system to cite this version : HAL Id: hal-01489782 Flexible Load-shedding using Gossip Communication in a Multi-Agents System. in 10th IEEE International Conference on Self-Adaptive and Self-Organizing Systems, 2017, pp. 1-10.

[48] Derakhshan G, Shayanfar HA, Kazemi A. The optimization of demand response programs in smart grids. Energy Policy, 2016; 94: 295-306.

[49] Nygard KE., Ghosn SB, Chowdhury M, Minhaz, Loegering D, McCulloch R, and Ranganathan P, "Optimization models for energy reallocation in a smart grid," 2011 IEEE Conf. Comput. Commun. Work. (INFOCOM WKSHPS), pp. 186-190, 2011.

[50] Chiu TC, Shih YY, Pang AC, and Pai CW. Optimized Day-Ahead Pricing with Renewable Energy Demand-Side Management for Smart Grids. IEEE Internet Things J., 2016; 4662c.: 1-1. 